\title{
OSCILLATION CRITERIA FOR ELLIPTIC SYSTEMS
}

\author{
W. ALLEGRETTO AND C. A. SWANSON
}

ABstract. Oscillation criteria are established for quasilinear elliptic partial differential systems of second order in unbounded domains of Euclidean space. The main departures from earlier investigations are: (1) systems of partial differential equations are considered; (2) the equations are nonlinear; (3) the matrices involved are not required to be positive definite; and (4) a direct method of the Calculus of Variations is used rather than the earlier majorization method.

1. Introduction. The first general oscillation criterion for partial differential equations was obtained by I. M. Glazman in 1958 [5]. This is a natural extension of the classical Kneser-Hille criterion to the Schrödinger equation $\nabla^{2} u+B(x) u=0\left(x \in R^{n}\right)$ and involves the limiting behavior of the minimum of $B(x)$ on the sphere $|x|=r$ as $r \rightarrow \infty$. An analogue of Glazman's theorem and other oscillation criteria were obtained recently for second order linear and quasilinear elliptic equations by Allegretto [1], [2], [3], Headley and Swanson [7], and Swanson [12]. Some extensions to elliptic equations of order $2 m$ also have been derived by a modification of Glazman's majorization method [4], [6].

The oscillation criteria cited above all involve either limits at infinity or integrals of suitable majorants (or minorants) of the coefficients of the differential operator. The results are derived by comparison of the given differential equation with a separable equation possessing a solution with an oscillatory radial function as a factor, and use of any of the well-established one dimensional oscillation criteria.

Our first purpose here is to develop oscillation criteria for partial differential equations which do not depend upon one dimensional criteria: We dispense with the majorization procedure altogether and use a direct method of the type used in the Calculus of Variations. Unlike all earlier results, our criteria are not one dimensional in character if $n>1$. Specialization to the case $n=1$ yields either a new oscillation criterion for ordinary differential equations (or systems) or an independent proof of a known result. Our criteria involve integrals

Received by the editors May 18, 1970.

AMS 1969 subject classifications. Primary 3511, 3547.

Key words and phrases. Quasilinear elliptic system, oscillation criterion, prepared matrix. 
of the actual coefficient functions appearing in the differential equation (rather than majorants) over the unbounded domain under consideration.

As a second departure from earlier investigations, we consider systems of partial differential equations. In the linear case, comparison theorems of Sturm's type for strongly elliptic systems were obtained by Kuks [10], Kreith [9], and Swanson [15]. Here we shall employ the matrix formulation of the system, and increase the generality by considering both the quasilinear case and inequalities for associated differential forms.

Oscillation criteria for matrix ordinary differential equations were obtained recently by Tomastik [16] and Swanson [14]. The oscillatory character of matrix solutions was established only for prepared matrices (also called conjugate matrices by some authors; in the linear case, the column vectors of a prepared matrix constitute a conjugate basis for the differential system in the language of $M$. Morse [11]). Indeed, easy examples show that the conclusions of the theorems in [14] and [16] are false without the hypothesis that the matrix solutions are prepared. Here we shall give a direct extension of the one dimensional results to $n$-dimensions without appealing to the former.

2. Definitions and notations. Oscillation criteria will be established for the matrix partial differential equation

$$
\begin{array}{r}
L V \equiv \sum_{i, j=1}^{n} D_{i}\left[A_{i j}(x) D_{j} V\right]+B\left(x, V, D_{1} V, \cdots, D_{n} V\right) V=0, \\
A_{i j}=A_{j i},
\end{array}
$$

and more generally for the inequality $V^{T} L V \leqq 0$ (as a form) in unbounded domains $G$ in $n$-dimensional Euclidean space $R^{n}$. As usual, points in $R^{n}$ will be denoted by $x=\left(x^{1}, \cdots, x^{n}\right)$ and differentiation with respect to $x^{i}$ by $D_{i}, i=1, \cdots, n$. The following assumptions will be made throughout:

Assumptions.

(1) $B, V$, and each $A_{i j}$ are $m$-square real matrix functions.

(2) Each $A_{i j}$ is symmetric and of class $C^{1}(G)$.

(3) The $m n$-square matrix $\left(A_{i j}(x)\right), i, j=1, \cdots, n$, is positive semidefinite in $G$.

(4) $B$ is symmetric, continuous, and bounded below (as a form) for $x \in G$ and for all values of the entries of $V$ and its partial derivatives.

The domain $\mathfrak{D}$ of $L$ is defined as the set of all $m$-square matrix functions $V \in C^{2}(G)$.

Let $M$ be a nonempty regular bounded domain within $G$, and let $F$ be the functional defined by 


$$
\begin{aligned}
F[u, V ; M]=\int_{M}\left[\sum_{i, j} D_{i} u^{T} A_{i j}(x) D_{j} u\right. & \\
& \left.-u^{T} B\left(x, V(x), D_{1} V(x), \cdots, D_{n} V(x)\right) u\right] d x
\end{aligned}
$$

for any piecewise $C^{1} m$-vector function $u$ on $M$ and any $y_{i}^{*} V \in \mathfrak{D}$.

A matrix $V \in C^{1}(G)$ is said to be prepared (or conjugate) iff $W_{i}(x)$ is symmetric for each $i=1, \cdots, n$, where

$$
W_{i}(x)=\sum_{j=1}^{n} V^{T}(x) A_{i j}(x) D_{j} V(x) .
$$

This definition is analogous to M. Morse's definition [11] of a conjugate basis for an ordinary linear system $(n=1)$, which has subsequently been modified and extended by Kuks [10], Tomastik [16], Swanson [14], and others.

The following natural extension of the well-known one dimensional definition of an oscillatory differential equation (or system) will be used in the sequel. The notation

$$
G_{a}=G \cap\left\{x \in R^{n}:|x|>a\right\}, \quad 0<a<\infty,
$$

will be used throughout.

Definition. The matrix differential inequality $V^{T} L V \leqq 0$ is termed (weakly) oscillatory in an unbounded domain $G \subset R^{n}$ iff every prepared solution $V$ of the inequality has the property that det $V(x)$ vanishes at some point of $G_{a}$ for all $a>0$.

This definition has been used by Kuks [10] in the linear case, by Kreith [8] when $m=1$, and by Tomastik [16] when $n=1$.

\section{Oscillation criteria.}

THEOREM 1. The inequality $V^{T} L V \leqq 0$ (as a form) is oscillatory in an unbounded domain $G$ if

(1) for arbitrary $a>0, G_{a}$ contains a nonempty regular bounded domain $M_{a}$; and

(2) there exists a piecewise $C^{1}$ vector function $u_{a}$ on $\bar{M}_{a}$ such that $u_{a}$ vanishes identically on $\partial M_{a}$ and $F\left[u_{a}, V ; M_{a}\right]<0$ for every differentiable matrix $V$ with det $V(x) \neq 0$ on $\bar{M}_{a}$.

Proof. Suppose to the contrary that $V^{T} L V \leqq 0$ is not oscillatory, i.e. there exists a positive number $a$ and a prepared solution $V$ of the inequality such that det $V(x) \neq 0$ in $G_{a}$. Then a unique solution $w(x)$ of $u(x)=V(x) w(x)$ exists in $G_{a}$ for every $m$-vector $u(x)$. The following 
identity, similar to that in $[13$, p. 188] is easily verified by differentiation for any piecewise $C^{1}$ vector function $u$ :

$$
\begin{aligned}
\sum_{i, j}\left(V D_{i} w\right)^{T} & A_{i j}(x) V D_{j} w+\sum_{i} D_{i}\left[u^{T} \sum_{j} A_{i j}(x)\left(D_{j} V\right) w\right] \\
= & \sum_{i, j} D_{i} u^{T} A_{i j}(x) D_{j} u-u^{T} B\left(x, V, D_{1} V, \cdots, D_{n} V\right) u \\
& +(V w)^{T}(L V) w+\sum_{i} w^{T}\left[W_{i}(x)-W_{i}^{T}(x)\right] D_{i} w
\end{aligned}
$$

where $W_{i}(x)$ is given by (2). Since $V$ is prepared, the last term on the right side is identically zero. Taking $u$ to be the vector function $u_{b}$ $(b>a)$ in the hypothesis (2) and integrating over $M_{b}$, we obtain the contradiction $F\left[u_{b}, V ; M_{b}\right] \geqq 0$.

The following result is an application of Theorem 1 in the case that $G$ coincides with $R^{n}$.

THEOREM 2. The inequality $V^{T} L V \leqq 0$ is oscillatory in $R^{n}$ if (in addition to the assumptions stated in \$2)

(1) the mn-square matrix $\left(A_{i j}(x)\right)$ is bounded above (as a form); and

(2) there exists a diagonal element $B_{k k}$ of $B$ such that

(4) $\lim _{a \rightarrow \infty} a^{1-n} \int_{S_{a}} B_{k k}\left[x, V(x), D_{1} V(x), \cdots, D_{n} V(x)\right] d x=+\infty$

for every differentiable matrix $V(x)$ with $\operatorname{det} V(x) \neq 0$ for all sufficiently large $|x|$, where $S_{a}$ denotes the ball $\left\{x \in R^{n}:|x|<a\right\}$.

Proof. The domain $M_{a}$ of Theorem 1 will be specialized to the annular domain

$$
M_{a b}=\left\{x \in R^{n}: a<|x|<b\right\}
$$

$(b>a+2)$. Define the piecewise $C^{1}$ vector function $u_{a b}$ on $\bar{M}_{a b}$ by $u_{a b}(x)=e_{k} \phi_{a b}(x)$, where $e_{k}$ is the unit vector with 1 in the $k$ th position and 0 elsewhere, and

$$
\begin{aligned}
\phi_{a b}(x) & =r-a & & \text { if } a \leqq r \leqq a+1, \\
& =1 & & \text { if } a+1<r \leqq b-1, \\
& =b-r & & \text { if } b-1<r \leqq b
\end{aligned}
$$

$(r=|x|)$. Clearly $M_{a b}$ is a regular bounded domain with $M_{a b} \subset G_{a}$, and $u_{a b}=0$ on $\partial M_{a b}$ for arbitrary $a$ and $b, a>0, b>a+2$. The conclusion will then follow from Theorem 1 if it can be shown that $F\left[u_{a b}, V ; M_{a b}\right]<0$ for sufficiently large $b$ and for every differentiable $V$ with det $V(x) \neq 0$ on $\bar{M}_{a b}$. 
To prove this, first observe that

$$
\begin{aligned}
\int_{M_{a b}} \sum_{i, j} D_{i} u_{a b}^{T} A_{i j}(x) D_{j} & u_{a b} d x \\
& \leqq \alpha \int_{M_{a b}} \sum_{i}\left(D_{i} \phi_{a b}\right)^{2} d x \\
& =\alpha \int_{M_{a b}}\left|\nabla \phi_{a b}\right|^{2} d x \\
& =\alpha n^{-1} \omega_{n}\left[(a+1)^{n}-a^{n}+b^{n}-(b-1)^{n}\right],
\end{aligned}
$$

where $\alpha$ is an upper bound on $\left(A_{i j}(x)\right)$ and $\omega_{n}$ denotes the area of the unit $(n-1)$-sphere. ( $\omega_{1}=1$ by convention.) Similarly

$$
\begin{aligned}
\int_{M_{a b}} u_{a b}^{T} B\left(x, V, D_{1} V,\right. & \left.\cdots, D_{n} V\right) u_{a b} d x \\
\geqq & \beta \omega_{n} \int_{a}^{a+1}(r-a)^{2} r^{n-1} d r \\
& +\int_{M_{a+1, b-1}} B_{k k}\left(x, V, D_{1} V, \cdots, D_{n} V\right) d x \\
& +\beta \omega_{n} \int_{b-1}^{b}(b-r)^{2} r^{n-1} d r
\end{aligned}
$$

where $\beta$ is a lower bound on $B\left(x, V, D_{1} V, \cdots, D_{n} V\right)$. Subtracting (6) from (5) we find that

$$
\begin{aligned}
b^{1-n} F\left[u_{a b}, V ; M_{a b}\right] \leqq & b^{1-n}[g(a)+h(b)] \\
& -b^{1-n} \int_{M_{a+1, b-1}} B_{k k}\left(x, V, D_{1} V, \cdots, D_{n} V\right) d x,
\end{aligned}
$$

where $g(a)$ is independent of $b$ and $b^{1-n} h(b)$ is bounded in $2 \leqq b<\infty$. However, the last term on the right side of (7) has limit $-\infty$ as $b \rightarrow \infty$ by the hypothesis (4). Hence $F\left[u_{a b}, V ; M_{a b}\right]<0$ for sufficiently large $b$, and the conclusion follows from Theorem 1 .

Theorem 2 remains true if (4) is replaced by the obviously stronger condition

(8) $\lim _{a \rightarrow \infty} a^{1-n} \int_{S_{a}} \operatorname{tr} B\left[x, V(x), D_{1} V(x), \cdots, D_{n} V(x)\right] d x=+\infty$

where $\operatorname{tr} B$ denotes the trace of $B$. In the special case that $B$ is positive definite, (4) can be replaced by the equivalent condition 


$$
\lim _{a \rightarrow \infty} a^{1-n} \int_{S_{a}} \lambda\left[x, V(x), D_{1} V(x), \cdots, D_{n} V(x)\right] d x=+\infty
$$

where $\lambda$ denotes the largest eigenvalue of $B$.

Theorem 2 requires neither $\left(A_{i j}\right)$ nor $B$ to be positive definite. The positive semidefiniteness of $\left(A_{i j}\right)$ is essential, but an analogue of Theorem 2 can be obtained without the assumptions that $B$ is bounded below and $\left(A_{i j}\right)$ is bounded above. If $n=1$, conditions (4), (8), and (9) reduce to those given recently by Tomastik [16] and Swanson [14].

\section{REFERENCES}

1. W. Allegretto, Eigenvalue comparison and oscillation criteria for elliptic operators, J. London Math. Soc. (to appear).

2. - A comparison theorem for nonlinear operators, Ann. Scuola Norm. Sup. Pisa (to appear).

3. - Comparison and oscillation theorems for elliptic equations, Ph.D. Thesis, The University of British Columbia, April, 1969.

4. - Oscillation criteria for 4 th order elliptic equations, Boll. Un. Mat. Ital. 3 (1970), 357-361.

5. I. M. Glazman, On the negative part of the spectrum of one-dimensional and multi-dimensional differential operators on vector-functions, Dokl. Akad. Nauk SSSR 119 (1958), 421-424. (Russian) MR 20 \#4674.

6. V. B. Headley, Elliptic equations of order $2 m$, J. Math. Anal. Appl. 25 (1969), 558-568. MR 38 \#3565.

7. V. B. Headley and C. A. Swanson, Oscillation criteria for elliptic equations, Pacific J. Math. 27 (1968), 501-506. MR 38 \#797.

8. Kurt Kreith, Oscillation theorems for elliptic equations, Proc. Amer. Math. Soc. 15 (1964), 341-344. MR 33 \#6098.

9. - A Sturm theorem for strongly elliptic systems and applications, Bull. Amer. Math. Soc. 75 (1969), 1025-1027.

10. L. M. Kuks, Sturm's theorem and the oscillation of solutions of strongly elliptic systems, Dokl. Akad. Nauk SSSR 142 (1962), 32-35=Soviet Math. Dokl. 3 (1962), 24-27. MR 26 \#441.

11. M. Morse, $A$ generalization of the Sturm separation and comparison theorems in n-space, Math. Ann. 103 (1930), 52-69.

12. C. A. Swanson, An identity for elliptic equations with applications, Trans. Amer. Math. Soc. 134 (1968), 325-333. MR 38 \#400.

13. - Comparison and oscillation theory of linear differential equations, Mathematics in Science and Engineering, vol. 48, Academic Press, New York, 1968.

14. - Oscillation criteria for nonlinear matrix differential inequalities, Proc. Amer. Math. Soc. 24 (1970), 824-827.

15. - Comparison theorems for elliptic differential systems, Pacific J. Math. 33 (1970), 445-450.

16. E. C. Tomastik, Oscillation of nonlinear matrix differential equations of second order, Proc. Amer. Math. Soc. 19 (1968), 1427-1431. MR 38 \#372.

The University of British Columbia, Vancouver, British Columbia, Canada 